\title{
Thermal Unimolecular Elimination of Water from tert-Butyl Alcohol: Deuterium Kinetic Isotope Effects, Transition Structure, Reaction Path, and Mechanism
}

\author{
Bansi L. Kalra ${ }^{\ddagger}$ and David K. Lewis* \\ Department of Chemistry, Connecticut College, New London, Connecticut 06320 \\ Stephanie R. Singer, Anuradha S. Raghavan, and John E. Baldwin* \\ Department of Chemistry, Syracuse University, Syracuse, New York 13244 \\ B. Andes Hess, Jr.* \\ Department of Chemistry, Vanderbilt University, Nashville, Tennessee 37205 \\ Received September , 2004
}

Supporting Information: Geometries, total energies and zero point energy corrections for TBA, TBATS, and TS (4 pages). 


\section{TBA}

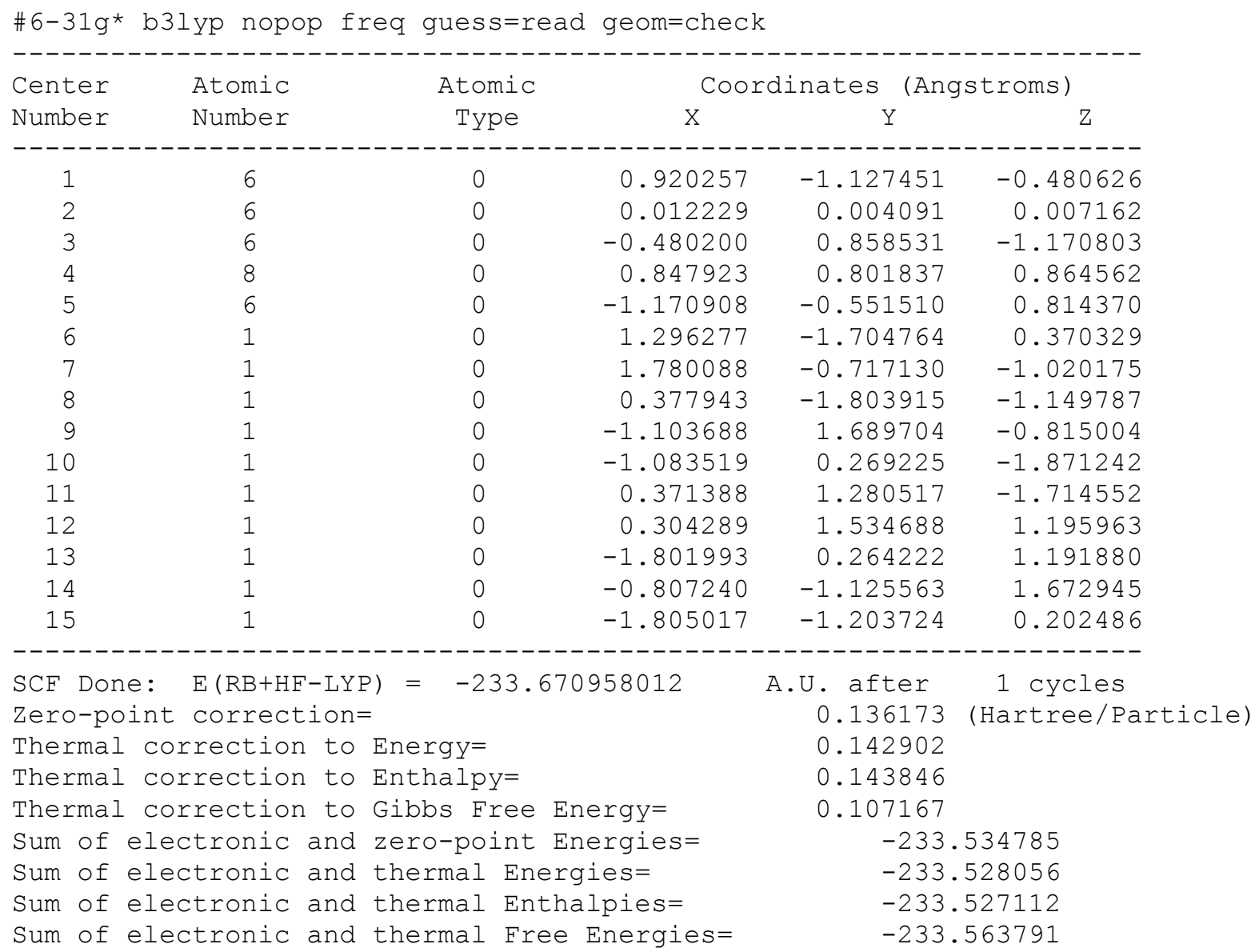




\section{TBATS}

\begin{tabular}{|c|c|c|c|c|c|}
\hline \multicolumn{6}{|c|}{ \#6-31g* b31yp nopop freq } \\
\hline Center & Atomic & Atomic & \multicolumn{3}{|c|}{ Coordinates (Angstroms) } \\
\hline Number & Number & Type & $\mathrm{X}$ & Y & Z \\
\hline \multicolumn{6}{|c|}{------------------------ - - - - - - - - - - - - - - - - - - - - - - - - - - - - - - - - - - - - - } \\
\hline 1 & 6 & 0 & -0.022812 & 1.517136 & 0.283356 \\
\hline 2 & 6 & 0 & -0.004639 & -0.028641 & 0.235413 \\
\hline 3 & 6 & 0 & -1.247513 & -0.577835 & -0.481125 \\
\hline 4 & 8 & 0 & -0.028992 & -0.451614 & 1.613312 \\
\hline 5 & 6 & 0 & 1.279236 & -0.549789 & -0.427856 \\
\hline 6 & 1 & 0 & 0.866135 & 1.952103 & -0.184662 \\
\hline 7 & 1 & 0 & -0.048447 & 1.839737 & 1.327454 \\
\hline 8 & 1 & 0 & -0.900696 & 1.932495 & -0.221909 \\
\hline 9 & 1 & 0 & -1.247803 & -1.676010 & -0.480255 \\
\hline 10 & 1 & 0 & -1.286500 & -0.246719 & -1.525497 \\
\hline 11 & 1 & 0 & -2.156281 & -0.235779 & 0.025238 \\
\hline 12 & 1 & 0 & -0.018280 & -1.422226 & 1.616455 \\
\hline 13 & 1 & 0 & 1.303787 & -1.647720 & -0.426453 \\
\hline 14 & 1 & 0 & 2.157974 & -0.187897 & 0.116226 \\
\hline 15 & 1 & 0 & 1.354843 & -0.217407 & -1.469818 \\
\hline \multicolumn{6}{|c|}{---------------------------------------------------------------------- } \\
\hline \multicolumn{6}{|c|}{ SCF Done: $\quad E(R B+H F-L Y P)=-233.665866283 \quad A}$. \\
\hline \multicolumn{6}{|c|}{ 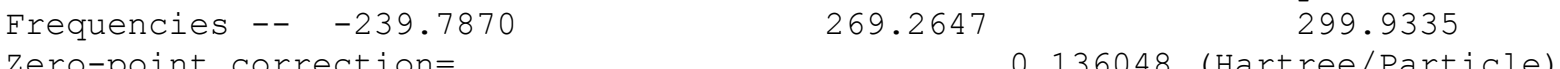 } \\
\hline \multirow{2}{*}{\multicolumn{6}{|c|}{$\begin{array}{lll}\text { Zero-point correction }= & 0.136048 \\
\text { Thermal correction to Energy }= & 0.142095\end{array}$}} \\
\hline & & & & & \\
\hline \multirow{2}{*}{\multicolumn{3}{|c|}{$\begin{array}{l}\text { Thermal correction to Enthalpy= } \\
\text { Thermal correction to Gibbs Free }\end{array}$}} & \multicolumn{3}{|c|}{0.143039} \\
\hline & & & Thermal correction to Gibbs Free Energy= & \multicolumn{2}{|l|}{0.107609} \\
\hline \multicolumn{4}{|c|}{ Sum of electronic and zero-point Energies= } & \multicolumn{2}{|c|}{-233.529818} \\
\hline \multicolumn{4}{|c|}{ Sum of electronic and thermal Energies= } & \multicolumn{2}{|c|}{-233.523771} \\
\hline \multicolumn{4}{|c|}{ Sum of electronic and thermal Enthalpies= } & \multicolumn{2}{|c|}{-233.522827} \\
\hline \multicolumn{4}{|c|}{ Sum of electronic and thermal Free Energies= } & \multicolumn{2}{|c|}{-233.558257} \\
\hline
\end{tabular}




\section{TS}

\begin{tabular}{|c|c|c|c|c|c|}
\hline \multicolumn{6}{|c|}{ \#6-31g* b3lyp nopop freq } \\
\hline Center & Atomic & Atomic & \multicolumn{3}{|c|}{ Coordinates (Angstroms) } \\
\hline Number & Number & Type & $\mathrm{X}$ & Y & Z \\
\hline \multicolumn{6}{|c|}{------------------------ - - - - - - - - - - - - - - - - - - - - - - - - - - - - - - - - - - - - - } \\
\hline 1 & 6 & 0 & 1.206650 & -0.484833 & -0.727905 \\
\hline 2 & 6 & 0 & -0.090118 & -0.294113 & -0.162384 \\
\hline 3 & 6 & 0 & -1.194611 & 0.295288 & -0.997589 \\
\hline 4 & 6 & 0 & -0.497971 & -1.071625 & 1.057983 \\
\hline 5 & 1 & 0 & 1.819153 & -1.289413 & -0.324631 \\
\hline 6 & 1 & 0 & 1.322952 & -0.302216 & -1.795349 \\
\hline 7 & 1 & 0 & -1.955963 & 0.789246 & -0.387512 \\
\hline 8 & 1 & 0 & -1.682938 & -0.520126 & -1.550873 \\
\hline 9 & 1 & 0 & -0.804871 & 1.002106 & -1.736038 \\
\hline 10 & 1 & 0 & -1.286743 & -0.567474 & 1.621521 \\
\hline 11 & 1 & 0 & 0.357132 & -1.237915 & 1.717361 \\
\hline 12 & 1 & 0 & -0.875000 & -2.053528 & 0.735748 \\
\hline 13 & 8 & 0 & 0.607803 & 1.334534 & 0.790710 \\
\hline 14 & 1 & 0 & 0.317261 & 2.172073 & 0.383850 \\
\hline 15 & 1 & 0 & 1.383347 & 0.662949 & -0.010513 \\
\hline \multicolumn{6}{|c|}{-----------------------------------------------------------------------} \\
\hline \multicolumn{6}{|c|}{ SCF Done: $\quad E(R B+H F-L Y P)=-233.562087937 \quad A}$. \\
\hline \multicolumn{6}{|c|}{ Frequencies -- -1948.8459 211.8284} \\
\hline \multicolumn{6}{|c|}{ Zero-point correction $=\quad 0.128364$ (Hartree/Particle) } \\
\hline \multicolumn{4}{|c|}{ Thermal correction to Energy= } & 0.135206 & \\
\hline \multicolumn{4}{|c|}{ Thermal correction to Enthalpy= } & 0.136150 & \\
\hline \multicolumn{4}{|c|}{ Thermal correction to Gibbs Free Energy= } & 0.099190 & \\
\hline \multicolumn{4}{|c|}{ Sum of electronic and zero-point Energies= } & \multicolumn{2}{|c|}{-233.433724} \\
\hline \multicolumn{4}{|c|}{ Sum of electronic and thermal Energies= } & \multicolumn{2}{|c|}{-233.426882} \\
\hline \multicolumn{4}{|c|}{ Sum of electronic and thermal Enthalpies= } & \multicolumn{2}{|c|}{-233.425938} \\
\hline \multicolumn{4}{|c|}{ Sum of electronic and thermal Free Energies= } & \multicolumn{2}{|c|}{-233.462898} \\
\hline
\end{tabular}

